\title{
Prosthodontic rehabilitation of young patient with dentinogenesis imperfecta using complete overlay denture
}

\author{
Dr Ajay Sabane ${ }^{1}$, Dr Namrata Wadhwani ${ }^{2}$, Dr Darshit Doshi ${ }^{3}$, \\ Dr Harshwardhan Sawant ${ }^{4}$ Dr Seema Patil ${ }^{5}$ \\ ${ }^{\text {I}(D e p a r t m e n t ~ o f ~ P r o s t h o d o n t i c s, ~ B h a r a t i ~ V i d y a p e e t h ~ D e e m e d ~ U n i v e r s i t y ~ D e n t a l ~ C o l l e g e ~ a n d ~ H o s p i t a l, ~}$ \\ Pune, India) \\ ${ }_{2}^{2}$ (Department of Prosthodontics, Bharati Vidyapeeth Deemed University Dental College and Hospital, \\ Pune, India) \\ ${ }^{3}$ (Department of Prosthodontics, Bharati Vidyapeeth Deemed University Dental College and Hospital, \\ Pune, India) \\ ${ }^{4}$ (Department of Prosthodontics, Bharati Vidyapeeth Deemed University Dental College and Hospital, \\ Pune, India) \\ ${ }^{5}$ (Department of Prosthodontics, Bharati Vidyapeeth Deemed University Dental College and Hospital, \\ Pune, India)
}

\begin{abstract}
Prosthodontic rehabilitation of patient with dentinogenesis imperfecta is a challenging task. Treatment protocol depends according to clinical case. Various reports in literature provide some general guidelines for deciding treatment planning. The case report presented here describes a full mouth rehabilitation of a young patient with dentinogenesis imperfecta prosthodontically treated by maxillary and mandibular complete overlay dentures.
\end{abstract}

Keywords: Complete overlay denture, Dentinogenesis Imperfecta, Full Mouth Rehabilation,

\section{Introduction}

Dentinogenesis imperfecta is inherited as an autosomal dominant trait. In fact this disorder is one of the most common dominantly inherited disorders in humans.(1)What happens in dentinogenesis imperfecta is the scalloping which is normally present at the dentino-enamel junction which is believed to help the mechanical interlocking between the two dental hard tissues together are defective in these conditions which leads to fracture of enamel easily from the defective dentin.(2)The dentin exposed because of that may then undergo rapid and severe attrition. (3) It is necessary to establish early diagnosis along with proper treatment plan (4) (5) (6)

An overlay denture is a partial or complete removable denture which is fabricated over retained teeth or roots of the teeth that are not prepared with any kind of coping to interface with the denture.(7) This is in contrast to an conventional over-denture in which usually the remaining teeth require endodontic treatment and cast gold or other metal copings.(8) Indications for an overlay denture include congenital disorders (e.g., cleft palate), apart from that oligodontia, ectodermal dysplasia, dentinogenesis imperfecta and cleido-cranial dysplasia. The fabrication of such type of overlay dentures may also be used as a treatment of choice for other disorders that are associated with missing or malformed teeth, and for patients with severely abraded or eroded teeth.(9)

\section{Case Report}

A 9 year old male patient came to Department Of Prosthodontics, Bharati Vidyapeeth Deemed University Dental College And Hospital, Pune with a chief complaint of difficulty in eating and compromised aesthetics. Patient had experienced continuous chipping of teeth while mastication. The colour of primary dentition was yellowish. [Fig. 1\&2]

\section{Radiographic Examination}

OPG revealed partial obliteration of pulp chambers with abnormal pulpal morphology. No evidence of any periapical pathology with any tooth was revealed.

\section{Diagnosis}

After thorough examination and correlating clinical and radiographic findings, diagnosis of dentinogenesis imperfect was made. 
All the primary teeth were present with loss of coronal tooth structure. Intra-oral examination of patient showed loss of vertical dimension at occlusion. [ Fig. 3 ]

\section{Treatment Procedure}

- Primary impressions were made using irreversible hydrocolloid (Tropicalgin, Zhermack) and primary casts were made from these impressions.

- Spacer wax was adapted on both the primary casts and tissue stops were made (4 tissue stops in maxillary and 2 on mandibular arch) preferably on the head tissue area i.e on the area of the worn teeth.

- After adaptation of spacer wax, special trays were prepared using green tray compound. Trays were trimmed from periphery keeping the border $2 \mathrm{~mm}$ short of the sulcus.

- After checking the special tray in mouth Border moulding using green stick compound was done with proper border movements. [ Fig. 4] Following this procedure final impressions were made using medium body elastomeric impression material after removal of spacer wax from the special tray. [ Fig. 5 ]

- Master casts were made from these final impressions. [ Fig. 6 ] Maxillary and mandibular record bases with appropriate wax rims were made on this casts. A face-bow transfer and jaw relation records are taken to relate casts which are mounted on a suitable articulator. A class 2 relationship between mandible and maxilla was encountered. Following methods were used to achieve the VDO: inter-occlusal distance, phonetics, swallowing, facial appearance and patient preferences.

Since the patient was in transition period, consideration of teeth selection was difficult and was made in consultation with the patient and parent. "Bambino Tooth" (Major Dental) teeth set was selected for this patient.

- The selected denture teeth are arranged in wax and tried in patient's mouth for evaluation of function and aesthetics. Since the mandibular arch was comparatively smaller than the maxillary arch, in maxillary arch one premolar in both the quadrant was included while the teeth arrangement to orient class 1 molar relationship and proper aesthetics. [ Fig. 7 and 8 ]

- After satisfactory try-in the denture was processed, remounted to adjust the occlusion and then delivered to the patient. During final try-in, the denture is seated and occlusal and flange portions of the denture were adjusted.

Home care instructions were given which include removal of dentures at night and brushing both the dentures and natural teeth after every meal.

\section{Advantages of Overlay Dentures}

Complete overlay denture has numerous advantages for the paediatric patient. Apart from improved mastication and aesthetics of the patient, speech also may be improved. The alveolar bone is still being maintained by the retention of teeth compared to its loss when teeth are extracted. With the retained roots of the teeth and their periodontal ligament, there is improved proprioception as compared to a complete denture that is fabricated over an edentulous ridge.(10) Compared to an overdenture, an overlay denture is cost effective, requires less chairside time, and does not require any specialized tooth preparation. When fabricated properly, an overlay denture has good support with excellent retention and stability. Possibly, the most important advantage of an overlay denture is the positive psychological support for the child.

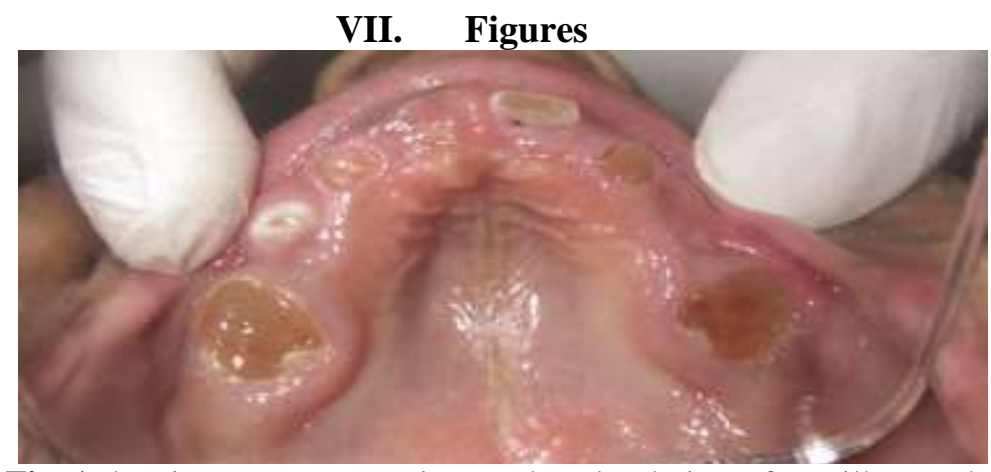

Fig. 1 showing pre-treatment intraoral occlusal view of maxillary arch 


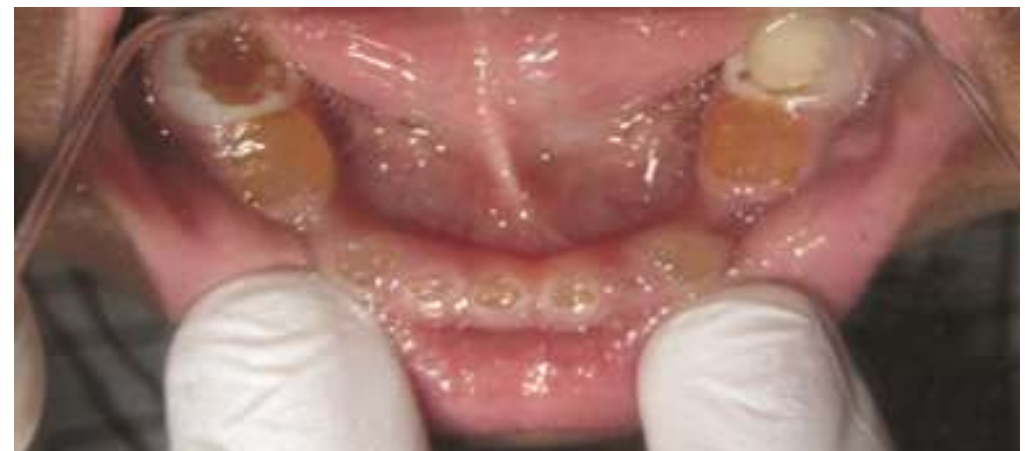

Fig. 2 showing pre-treatment intraoral occlusal view of mandibular arch

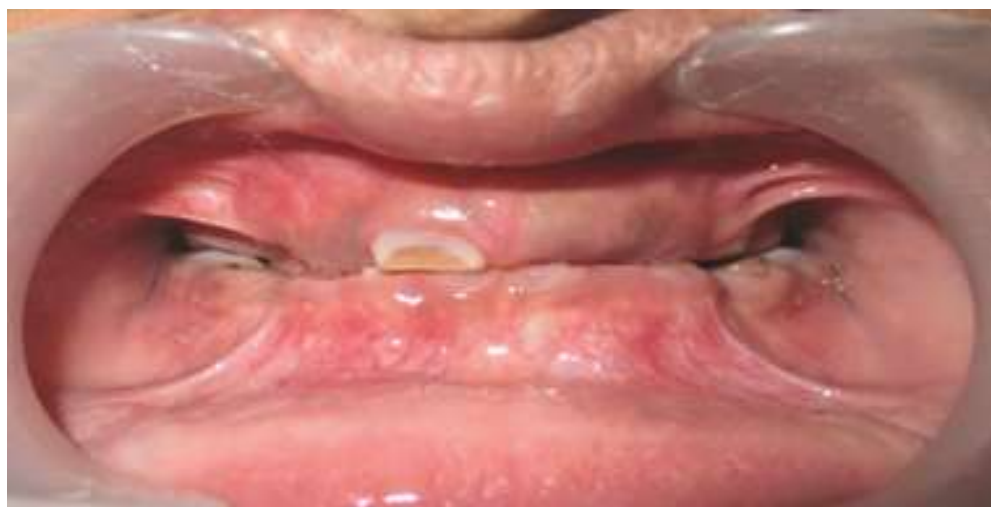

Fig. 3 showing intraoral anterior view of a patient presenting severely worn maxillary and mandibular dentition and loss of VDO.

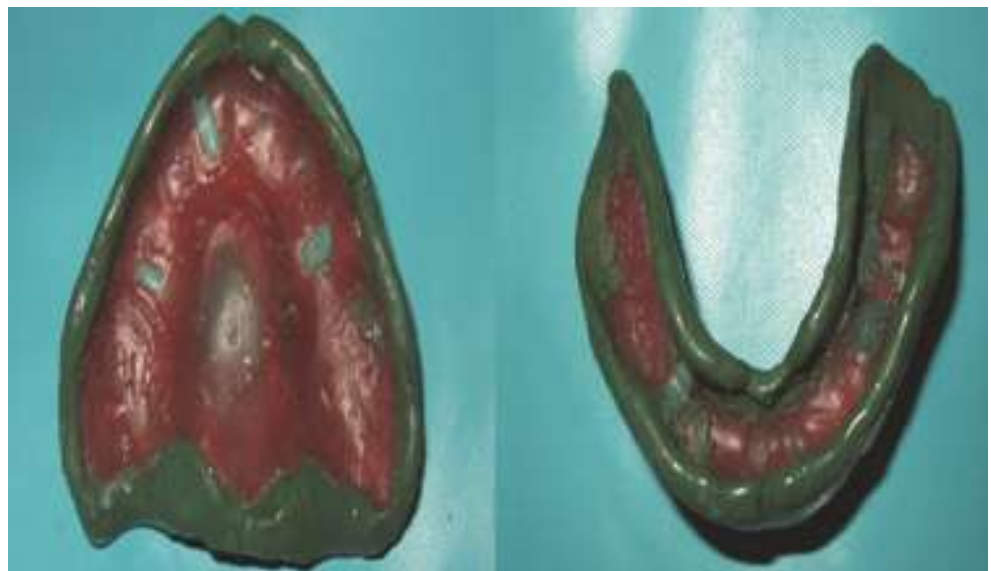

Fig. 4 showing border moulding done on maxillary and mandibular special trays

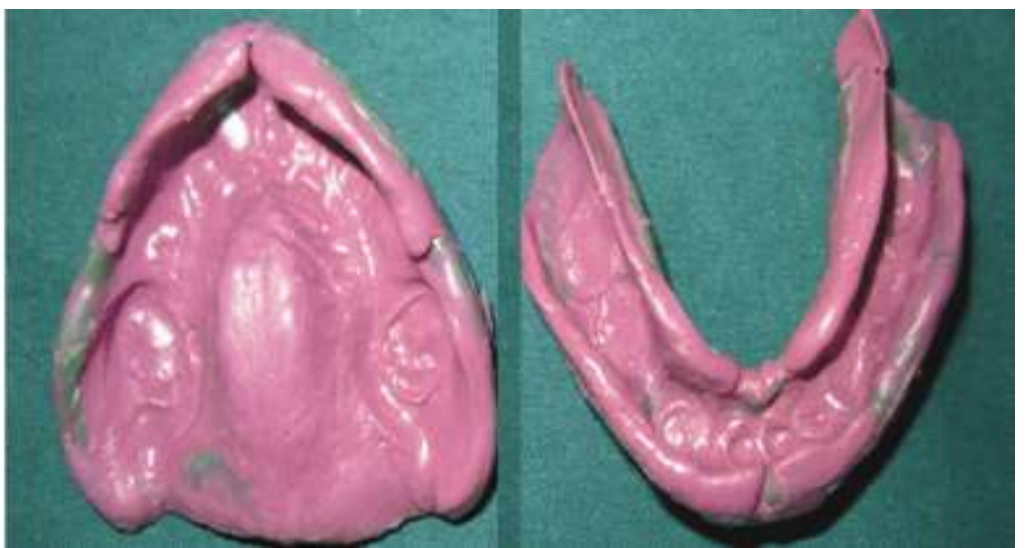

Fig. 5 showing final impression made using medium body elastomeric impression material 


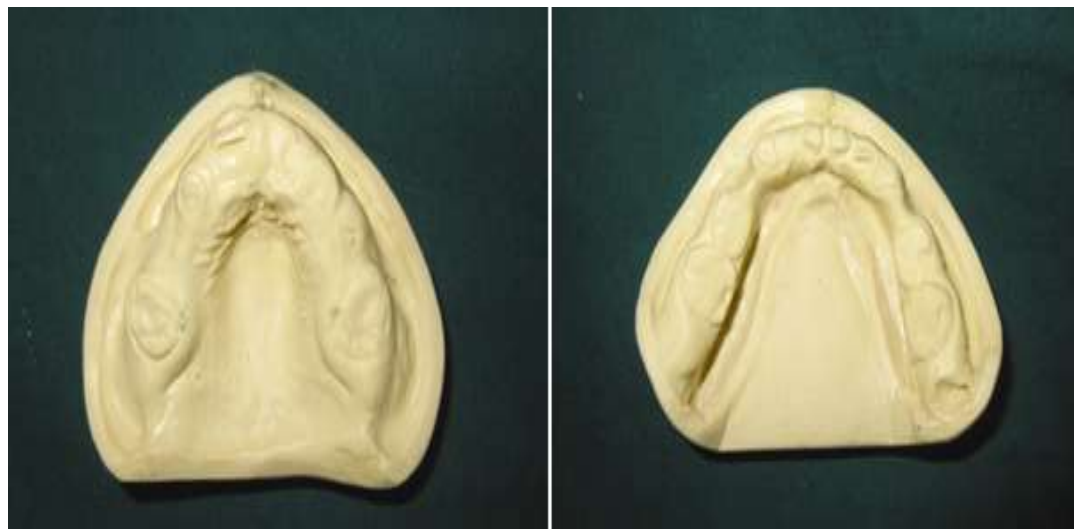

Fig. 6 showing master cast
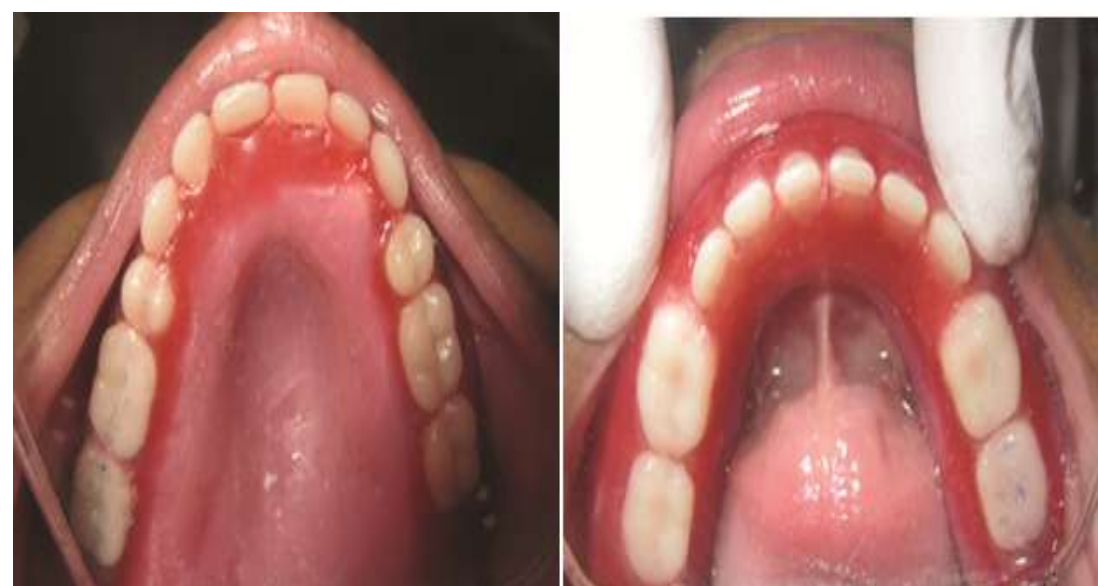

Fig. 7 showing intr-oral occlusal view of arrangement of teeth in maxillary and mandibular arch

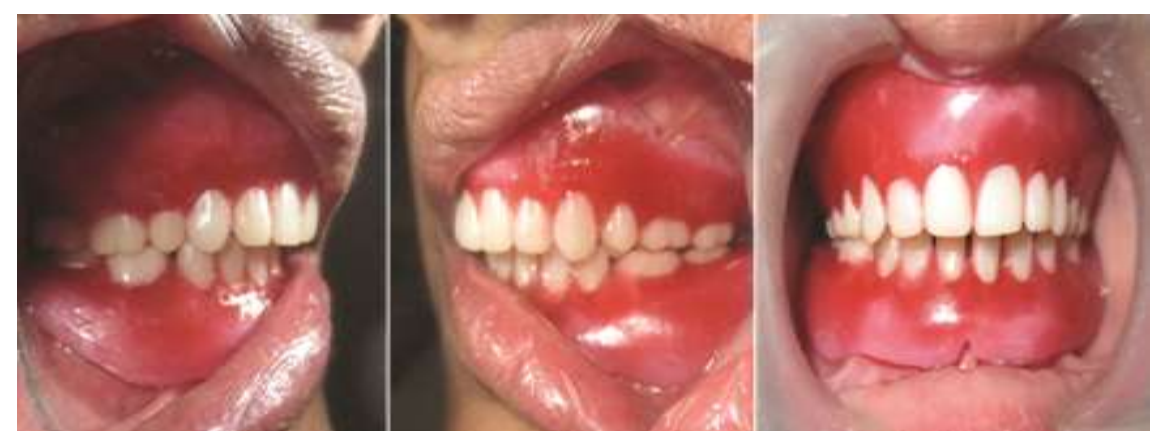

Fig. 8 showing intraoral views and inter-occlusal relationship between two arches.
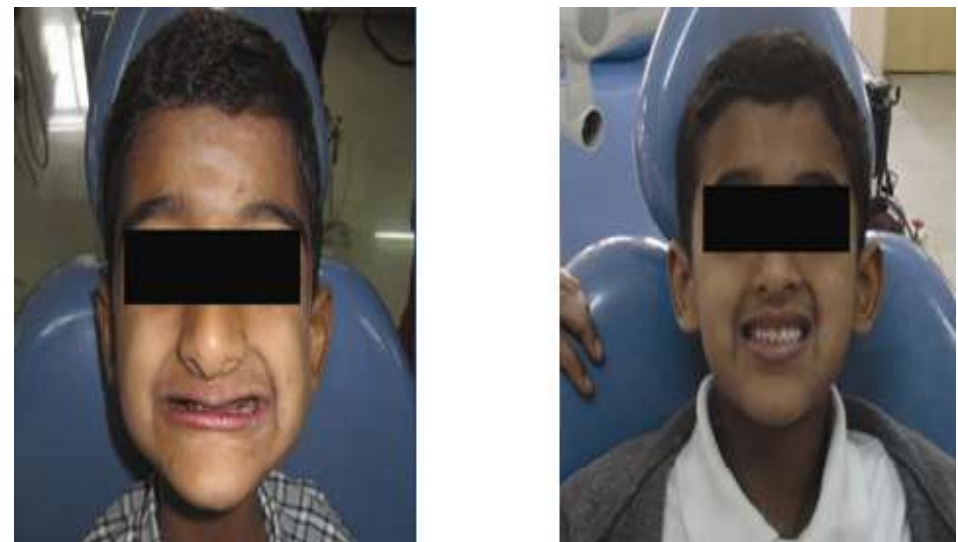

Fig. 9 showing pre-operative and post operative view of the patient. 


\section{Conclusion}

One of the greatest challenges for the prosthodontist is to provide adequate treatment to achieve functional and aesthetic restoration in cases of disease like dentinogenesis imperfecta. Early diagnosis and treatment are essential for obtaining a favourable prognosis. To preserve existing tissues and rehabilitation of the function and aesthetic with a multidisciplinary approach is of utmost importance. Prosthodontic rehabilitation of this kind greatly improves function, aesthetics and proves to be great psychological boost to the patient's well-being.

\section{References}

[1]. Bouvier D, Duprez JP, Morrier JJ, Bois D. Strategies for rehabilitation in the treatment of dentinogenesis imperfecta in a child: a clinical report. J Prosthet Dent. 1996;75:238-41.

[2]. Takagi Y, Koshiba H, Kimura O, Kuboki Y, Sasaki S. Sasaki. Dentinogenesis imperfecta: Evidence of qualitative alteration in the organic dentin matrix. J Oral Pathol. 1980;9:201-9.

[3]. Pettiette MT, Wright JT, Trope M. Dentinogenesis imperfecta: Endodontic implications. Oral Surg Oral Med Oral pathol Oral Radiol Endod

[4]. Sutherland EP, Smith CJ. The teeth in Osteogenesis and Dentinogenesis imperfecta. Br Dent J. 1980;149:287-9.

[5]. Witkop CJ., Jr Hereditary defects of dentin. Dent Clin North Am. 1975;19:25-45.

[6]. Sheilds ED, Bixler D, EL-Kafrawy AM. A proposed classification for heritable human dentine defects with a description of a new entity. Archs Oral Biol. 1973;18:543-53.

[7]. Brewer, A., Fenton, A. The Overdenture. Dent. Clin. North Am. 1973;17:723-746.

[8]. Lord,J.L.,Teel 1969 The Over-dentures.Dental Clin North America 13(4),871-881

[9]. Johnson A, Winstanley RB: Use of simple overdentures in the treatment of young patients with developmental anomalies. Quintessence 11: 27-33, 1987

[10]. Robert J Crum, R J Loiselle. Oral perception and proprioception: A review of literature and its significance to Prosthodontics. J Prosthet Dent, 1972;28;215-230 